\title{
Enhancing Security Features for IoT Devices by Integration with Block Chain Technology
}

\author{
B.Venkatesh, Ch. Srinivasa Reddy, Ch.V.Bhargavi
}

\begin{abstract}
System of bodily hubs or "matters" joined with hardware, programming, sensors, and linked to enact articles to move information from servers, included frameworks, or doubtlessly distinctive associated devices depending on a numerous correspondence foundations may be actualized with net of factors (IoT) version. IoT facts gathered from various sensors, hubs and government are moved to the cover over the net. The principle target of IoT protection is to ensure thriller of the statistics, and make certain the assurance of the consumer's statistics, frameworks, software program's facts substance, and smart machine's of the IoT, via manner of ensures the administrations accessibility of IoT organic gadget. The number one purpose of this exam article is to enhance protection highlights to IoT device becoming a member of with rectangular chain. The significance of Bit-coin the usage of rectangular chain innovation, which changed into at that factor set up for a few, financial nicely well worth exchanges because it have been. Anyhow, because of its Non-delivered collectively engineering, agile corruption and cryptographic defend benefits, for instance, pseudonymous personalities, statistics trustworthiness and take a look at, scientists and safety professionals round the sector are concentrating on the rectangular chain to decide coverage and protection issues of IoT. In this article, we have positioned a few right down to earth problems which can be associated with the becoming a member of of IoT devices with the square chain. At closing, we endorse a course ahead to determine a part of the large difficulties to the rectangular chain's utilization in IoT based totally software program.
\end{abstract}

Index Terms-IoT, Sensors, Block Chain, Integration

\section{INTRODUCTION}

There was intense interest and advancement within the internet of factors (IoT) based totally administrations round the arena, specially in wellness division, administrations and application introduction and in especially thick areas for the usage of IoT. It's far required to enroll in billions of gadgets by 2020 [1]. Global's financial system and individuals' existence may be improved by way of the usage of IoT. Preference is to make approximately USD 7.1 trillion commitments to the worldwide financial system through 2020 [2]. Be that as it may, within the meantime, IoT devices are unprotected because of great security highlights slips by simply as clients' protection worried, that are known to the designers but security in IoT devices is both disregarded or dealt with as an addendum [3].

It's far run of the mill for the eventual destiny of IoT that its sensible model is restored from steeply-priced, common and over-curved integrated layout to an automated and selfguided decentralized model, this type of transformation will

Revised Manuscript Received on April 12, 2019.

B.Venkatesh, Department of CSE, Vignan's Institute of Information Technology Duvvada, Visakhapatnam, A.P, India.

Ch. Srinivasa Reddy, Department of CSE, Vignan's Institute of Information Technology Duvvada, Visakhapatnam, A.P, India.

Ch.V.Bhargavi, Department of CSE, Vignan's Institute of Information Technology Duvvada, Visakhapatnam, A.P, India. give wide scope of usage, low foundation price, independence for devices, at ease sports in a trustless state of affairs, customer driven safety, get to manipulate and extra towards gadget assaults.

Square chain is being considered as one of the realistic technique to renowned required decentralization and offers structures that are disgraceful in such way [4].

Notwithstanding the fact that rectangular chain changed into before everything considered as a economic trade conference as Bit coin, however due to its cryptographic protection advantages, as an example, pseudonymous personalities (IDs), decentralization, adaptation to nonimportant failure, change honesty and affirmation, professionals and protection investigators around the world are concentrating at the square chain to decide protection and safety problems of identified with IoT.

In spite of the reality that an average Bit coin square chain confinements are, as larger diploma of usage, delay occurred within the change take a look at and large sparing capacity, spillage construe for protection and basic degree count and the best desires, that square chain innovation must be investigated profoundly earlier than it has a tendency to be performed properly and ably in an IoT associated software's.

\section{PREFERRED CONCEPT OF IOT}

We centered on giving a brief depiction of the IoT innovation on this level,

\section{A. IoT Devices}

In earlier trade it became stated, the IoT will cover severa facts association systems wherein the hubs are conveying utilizing net with every other. "

It's alluded as both Node or .substances within the workplaces are typically called as utility devices, they displayed severe traits. [5].

a. Identity: every IoT system need to display an inexpensive strong point, just like an internet Protocol rendition 6 (IPv6) cope with for you to talk amongst all objects [6].

b. Sensing: The detecting strategies are actualized to secure statistics from the modern-day actuators around the hubs correspondence [26].

c. Communication: communique implies the between connection strategies which can be applied for you to companion with all hubs using items with each different [6].

d. Computation: The calculation structures are gotten to take a shot on the records that is procured from the hubs [7]. 
e. Offerings: utility administrations are activities which produces for the hubs supporters in a concurrence with the statistics which they were given from the genuine running detecting area [7].

f. Semantics: The hubs within the IoT have the restriction stage to get the first-rate possible hub facts from place of business at that factor to deliver this software content as administrations at the required time [8].

\section{PROTECTION MEASUREMENTS INSIDE THE IOT}

Beyond to finding the possible wellness dangers in the IoT model, at first we must find out the associated protection related guarantee requirements. After exceptional examinations have completed and closed the assurance necessities for IoT [9, 10]. Resulting set of shield requirements have been proposed utilizing the above estimations for IoT.

A. Confidentiality: This expression clarifies interconnected settings. Within the first region, clarified approximately unlawful responsibilities should not suitable to utilize it for person records. Besides, its firmly pronouncing about the defend of categorized and character facts.

B. Integrity: Integrity says that facts and the IoT hubs can't be changed or utilized, through illicit customers and objects.

C. Availability: Availability clarifies that the processing assets and substance ought to be open at whatever factor they're required by an software management. this implies the IoT devices which can be applied to realize the genuine air the registering gadgets which can be used to stack and building up the utility content and the shrewd channels must work correctly.

D. Authenticity: Authenticity affirms that the utility substance and tasks are valid. Obviously, this standard ought to approve that the gatherings that consist of in an pastime ought to be those whom they assure to be.

\section{SAFETY TROUBLES IN THE IOT}

The assurance in IoT is characterized by means of pinnacle precedence ponder fixation considering the fact that it's far an advancement of the ordinary, unprotected internet model wherein the institutions in the virtual global gain the real global. Particularly, the security strategies in the IoT want to talk to the normal systems administration affects and within the interim, they bring to the desk secure institutions for the two forms of members of the family: person to-device and system to-character. So as to finish the previously mentioned guarantee necessities and distinguish appropriate treatment options, the accompanying issues should be tended to.

A. Interoperability: The improvement and the utilization of guarantee strategies in the IoT must not usually restrict the functional qualities of the IoT hubs.

B. Aid restrictions: The devices inside the IoT are organized via limited belongings in memory and calculation; subsequently, they may not bolster the profitable administrations of the commonplace health safety measures, as an example, the lopsided encryption.

C. Resilience to bodily attacks and cataclysmic events: The IoT hubs are inherently little with skinny or no wellbeing. As an example, a phone or a sensor machine will be removed, and the rigid hubs may be moved or harmed through catastrophic activities.

D. Autonomic control: The conventional application content material gadgets need the clients to broaden them. But, the IoT hubs need to set up their institutions together.

E. statistics volume: more than one IoT packages, for example, the savvy framework and keen metropolis process a huge quantity of worthwhile and character substance, which is a tough aim of a developing measure of protection dangers.

F. privacy security: normally, the IoT hubs contain important substance which have to be ensured and non-precise, recognizable and appendable.

G. Scalability: The IoT arranges normally take an interest in a massive range of articles. In this way, the safety and privacy defend strategies ought to not have the option to measure.

\section{GENERAL IDEA BLOCK CHAIN}

The Bit coin [11] has in all respects imaginatively changed the approach for procedure in financial really worth alternate arising brief on any outsider. The primary capacity of Bit coin is square chain. In simple vocabulary, rectangular chain contains an arrangement of squares in a way that every new square is cryptographically related to the former rectangular. Resulting from Bit coin, the squares keep a file of monetary exchange among Bit coin clients. Because of its in-built gain, for instance, permanence, evaluate ability, trade, unwavering first-rate and confirmation, blunder endurance, or more all sans consider process, rectangular chain is being presupposed to expect a fundamental process within the wellness of IoT organic device.

\section{A. Key ideas}

1. Transaction (TX): economic TX tactics are carried out utilising square chain stage, at the execution of a discretionary code as a notable agreement for [11]. In addition, due to an IoT running situations, TX might be a strategies for distributive patron or encompassing sensors' information.

2. Block: It is lots of TXs that took place in the slicing edge and have not been characterised at this point. The rectangular likewise has a square heading that holds, rectangular chain variant range, hash of the primary rectangular, an arbitrary nonce, time stamp and Merkle Root Hash of all the TXs built-in in the block. 
1. Block chain: It is a shared open ledger that keeps a data of all the TXs/blocks [12]. Vitalik Buterin in [13] gives another perspective that the essence of the block chain is informational and processual, and does not relate directly to the monetary sphere.

\section{CHALLENGES TO BLOCK CHAINS IMPEMENTATION IN IOT \& RESULTS}

To identify couple of genuine problems figuring out with rectangular chain's execution in IoT,

1. A Rpi-3 based totally sensor hub (situation 1) may be joined directly to the square chain as a total jump [14] or a mild square chain customer. A complete jump can verify additional TXs, but a mild client can just hold up a manner of its individual TXs.

2. The warm temperature sensor unearths the (person Interface) or a transportable (utility). The internet UI or transportable application joined to the rectangular chain jump drive the sensor examination to the square chain from begin to complete super know-how. As a result, a mobile or an internet application is the medium among IoT hubs and the rectangular chain.

3. In scenario 2 an IoT machine can be an asset restrained Arduino instrument or any extra established framework professional of honestly breaking down and speak the warmth sensor research statistics to a portal system.

4. The Arduino-based totally hub cooperates with the passage hub from first to ultimate slower and less sheltered far off transmission media. Further, this association likewise confines the rectangular chain primarily based hub to-hub contact [16], as now simply the entryway tool can ideal to make use of the square chain or wealthy contracts.

5. Much similar to in scenario 1, the passage likewise joins to the Geth hub via a web3 company and drives sensor information to the rectangular chain via an first rate understanding by way of techniques for an internet or a mobile application.

6. Aowever, there were clear problems recorded thru this plan. Proper off the bat, there's an inquiry of the way to make certain the sheltered contribution of sensor records to the rectangular chain? furthermore, at present, not one of the square chain degrees execute IoT-targeted TX validation legal guidelines and IoTbased accord conference. In conclusion, a center person most of the sensor gadget and the rectangular chain is the UI, which can't effect the cryptographic safety gotten by the rectangular chain. As an alternative, additional system, net, and alertness wellbeing safety measures should be taken into consideration.

\section{ASSOCIATED WORK}

There are some disbursed papers on IoT safety however not that bunches of pointed out the IoT well-being issued related to the prevailing fashion in IoT. In our examination, we have considered papers related to well being in IoT surroundings and its value is recovered via a web UI

machines, IoT improvement, notwithstanding verifying the net UI and cellular utility, IoT system consolidation with the square chain may be augmented by means of device enrolment, in which simply trendy hubs are worthy to compare with the rectangular chain and phone savvy knowledge techniques. in addition, savvy understandings can restrain admission to picked strategies to an exact gadget as it had been. Identifying with the physical assurance of IoT hubs, all of the unnecessary terminals like as JTAG and UART must be blocked.

As any open port can be used by a foe to enter the hub and fabricate malignant updates. Furthermore, a widespread wide variety of the open IoT hubs like as detecting machines do not have a secured execution foundation due to consumption impacts. For that reason, the system electricity check must over and over be decided to make sure its authenticity [15]. Beginning these days, the great majority of the IoT machines depending on the use of the cloud stage due to computational and ability deficiency in side figuring side and in mild of the equal, asset confinements IoT machines cannot be benefited as a fractional or whole gadget in a rectangular chain set-up. Along these lines, to ensure a sensitive changeover from cloud to square chain ward arrange, IoT machines can effect Fog Computing hardware that already are looking for after a few level of conveyance and are extra resourceful than IoT gadgets. The Fog hubs can work as square chain excavators and may inspire direct cooperation between IoT gadgets and the rectangular chain. The haze hubs can include rectangular chain little hubs to gather and mine the TXs extricated from the IoT hubs in a rectangular. The IoT hubs have adequate assets for be the overall gadgets. Alongside these traces, they can inventory up the rectangular chain and furthermore way and confirm the TXs. Alongside these strains, a big portion of the TXs from the IoT hubs could be transmitted to each the haze hubs.

Sooner or later, IoT can impact gift haze processing degree to execute rectangular chain equipment, until IoT hubs are added with set up rectangular chain mining usefulness to choose up at the maximum severe desired function of square chain's shared development.

\section{CONCLUSION}

No dithering, IoT is the expectancy of a loose digitized money related association of the globe by way of melting and customizing the enormous hubs. However, to get this function, it needs to revel in a hypothetical adjustment each at the association and the development phases of its safety highlights. The ones dates aren't all that lengthy, whilst innovation will cooperate with gear lacking of man or woman obstruction to reap execution capability degree, sturdiness, operational effectiveness, and cash related economic system. Alongside those strains, it is imperative to plot and increase an ensured rectangular chain based IoT framework that achieves the expectation of all requested a loose automated international. Similarly, execution viewpoints should likewise receive for due concept, in 
parallel to the well-being protection troubles. Henceforth, in this text, we basically presented about the IoT security threat inside the place of work, resultant for wellness and advanced requirements for IoT frameworks and key rectangular chain thoughts. We foreseen a manner supplied well being to IoT system fuse with the square chain.

\section{REFERENCES}

1. Q.okay.A. Mirza, G. Mohi-Ud-Din, I. Awan, A cloud-based totally vitality effective framework for upgrading the recognition and anticipation of modern malware, in: 2016 IEEE thirtieth worldwide convention on superior data Networking and packages (AINA), 2016, pp. 754-761. CransMontana.

2. F. Cadet, D.T. Fokum, coping with forswearing ofadministration attacks at the IP verbal exchange framework, in: SoutheastCon 2016, 2016, pp. 1-7. Norfolk, VA.

3. D.H. Sharma, C.A. Dhote, M.M. Potey, enforcing interruption the board as protection-as-an management from cloud, in: 2016 international conference on Computation device and statistics era for Sustainable solutions (CSITSS), 2016, pp.363-366. Bangalore.

4. Amos O. Olagunju, Farouk Samu, seeking out compelling nectar pot and nectar net frameworks for ongoing interruption discovery and counteractive action, in: complaints of the fifth Annual convention on research in statistics generation (RIIT '16), ACM, big apple, ny, united states, 2016, pp. 41-forty six.

5. M. Yevdokymenko, a flexible calculation for identifying and warding off attacks in media transmission systems, in: 2016 $1 / 3$ global medical-purposeful conference issues of info interchanges science and technology (\% S\&T), 2016, pp. a hundred seventy five-177. Kharkiv.

6. M. Portage, et al., A procedure to move Fail2ban information to a flexible endeavor interruption discovery and counteractive action framework, in: SoutheastCon 2016, 2016 , pp. 1-four. Norfolk, vol. A.

7. S. Vij, A. Jain, phone seizing: exam of interruption discovery and anticipation frameworks, in: 20160.33 global convention on Computing for Sustainable international development (INDIACom), 2016, pp. 2209-2214. New Delhi.

8. G. Kalnoor, J. Agarkhed, sample coordinating interruption discovery method for wireless Sensor Networks, in: 2016 2d global convention on Advances in electric, Electronics, facts, conversation and Bio-informatics (AEEICB), 2016, pp. 724728. Chennai.

9. S. Kumawat, A.ok. Sharma, A. Kumawat, Intrusion discovery and aversion framework making use of okay-gaining knowledge of grouping in cloud, in: 2016 third international convention on Computing for Sustainable global development (INDIACom), 2016, pp. 815-820. New Delhi.

10. A. Saracino; D. Sgandurra; G. Dini; F. Martinelli, "MADAM: a hit and proficient behavior based android malware popularity and aversion," in IEEE Transactions on reliable and comfy Computing, vol.PP, no.vol. ninety nine, pp.1-1.

11. S. Alsunbul, P. Le, J. Tan, B. Srinivasan, A system protection framework for detectingand forestalling potential hacking endeavors, in: 2016 international convention on facts Networking (ICOIN), 2016, pp. 449-454. Kota Kinabalu.

12. J. Filipek, L. Hudec, Securing transportable particularly appointed structures utilizing circulated firewall with PKI, in: 2016 IEEE fourteenth global Symposium on carried out system Intelligence and Informatics (SAMI), 2016, pp. 321325. Herlany.

13. A. Merlo, M. Migliardi, E. Spadacini, Balancing postponements and energy usage in IPS-empowered systems, in: 2016 thirtieth global conference on superior data Networking and applications Workshops (WAINA), 2016, pp. 267-272. Crans-Montana.
14. M. Chen, Y. Qian, J. Chen, ok. Hwang, S. Mao, L. Hu, privateness insurance and interruption evasion for cloudletbased healing statistics sharing, in: IEEE Transactions on Cloud Computing, 2007. In press.

15. P. Jokar, V. Leung, Intrusion discovery and avoidance for ZigBee-based totally domestic quarter organizes in intelligent matrices, in: IEEE Transactions on clever Grid, 2017. In press.

16. I. Indre, C. Lemnaru, Detection and avoidance framework towards digital assaults and botnet malware for facts frameworks and internet of factors, in: 2016 IEEE 12th global convention on intelligent computer conversation and Processing (ICCP), 2016, pp. a hundred seventy five-182. Cluj-Napoca.

\section{AUTHORS PROFILE}

Author-1 Photo

Author-2 Photo

Author-3 Photo
First Author personal profile which contains their education details, their publications, research work, membership, achievements, with photo that will be maximum 200-400 words.

Second Author personal profile which contains their education details, their publications, research work, membership, achievements, with photo that will be maximum 200-400 words

Third Author personal profile which contains their education details, their publications, research work, membership, achievements, with photo that will be maximum 200-400 words. 